\title{
Construction of a rabbit model with vinorelbine administration via peripherally inserted central catheter and dynamic monitoring of changes in phlebitis and thrombosis
}

\author{
LIQUAN HUANG ${ }^{1}$, GUIYUAN CHEN ${ }^{1}$, QINGHUA HU ${ }^{2}$, BO HU $^{3}$, LOUYING ZHU $^{4}$ and LUYAN FANG ${ }^{1}$ \\ ${ }^{1}$ Nursing Faculty, School of Medicine, Jinhua Polytechnic, Jinhua, Zhejiang 321007; ${ }^{2}$ Department of Orthopedics, \\ Jinhua Hospital of Traditional Chinese Medicine; ${ }^{3}$ Department of Obstetrics and Gynecology, Jinhua People's Hospital; \\ ${ }^{4}$ Jinhua Center of Laboratory Animals, Jinhua Food and Drug Inspection and Testing Institute, \\ Jinhua, Zhejiang 321000, P.R. China
}

Received July 7, 2021; Accepted November 10, 2021

DOI: $10.3892 / \mathrm{etm} .2022 .11135$

\begin{abstract}
Peripherally inserted central catheters (PICCs) are used for the administration of chemotherapy drugs, including vinorelbine. The present study aimed to construct a rabbit model with vinorelbine administration via PICC, and to dynamically monitor the formation of phlebitis and thrombosis. PICC was inserted into 48 rabbits following specific clinical procedures. The rabbits were randomly divided ( $n=6$ per group) into the following eight groups: i) Control (PICC in place for 1 day); ii) 2nd day of PICC placement (received the first cycle of vinorelbine administration); iii) 3rd day of PICC placement; iv) 7th day of PICC placement; v) 14th day of PICC placement; vi) 21st day of PICC placement; vii) 23rd day of PICC placement (received the second cycle of vinorelbine administration); and viii) 24th day of PICC placement. Hematoxylin and eosin staining was performed on catheter, ear vein and anterior vena specimens. Prothrombin time was measured using an automatic coagulation analyzer, followed by routine blood tests. Serum levels of inflammation- and thrombosis-related factors, including C-reactive protein, D-dimer, interleukin-2, interleukin-6, P-selectin and E-selectin, were measured using ELISAs. X-ray examination confirmed that the rabbit model with vinorelbine administration via PICC was successfully constructed. On the 1st and 23rd day of PICC placement,
\end{abstract}

Correspondence to: Professor Liquan Huang, Nursing Faculty, School of Medicine, Jinhua Polytechnic, 1188 Wuzhou Street, Jinhua, Zhejiang 321007, P.R. China

E-mail: huangliquan126@163.com

Abbreviations: PICC, peripherally inserted central catheter; CRP, C-reactive protein; D2D, D-dimer; IL-2, interleukin-2; IL-6, interleukin-6; ELISA, enzyme-linked immunosorbent assay; $\mathrm{H} \& \mathrm{E}$, hematoxylin and eosin

Key words: PICC, vinorelbine, catheter-related thrombosis, inflammation, prothrombin time thrombosis was observed in the catheter. Furthermore, on the 1st day of PICC placement, thrombosis was clearly observed in the ear vein and anterior vena samples. After vinorelbine administration, phlebitis occurred in the ear vein and anterior vena cava samples. With increasing time after vinorelbine administration via PICC, thrombosis and phlebitis were notably ameliorated. Moreover, on the day of vinorelbine administration, prothrombin time was significantly decreased and the serum levels of inflammation- and thrombosis-related factors were significantly increased compared with previous days. Collectively, the present study observed the formation and specific evolution of phlebitis and venous thrombosis after vinorelbine administration, providing a reference for the early prediction, timely prevention and treatment of PICC-related chemotherapy complications.

\section{Introduction}

Peripherally inserted central catheters (PICCs) have been widely applied for the administration of chemotherapy drugs (1-3). PICCs have the advantages of convenient operation, high safety, long indwelling time and low maintenance difficulty (4), and can provide patients with intravenous treatment for 7 days to 1 year, which is beneficial to reduce damage to blood vessels and avoid patient suffering associated with repeated punctures (5). Furthermore, PICC provides good venous access for nutrition and chemotherapy in patients with cancer (6). However, previous studies have indicated that PICC application has a significant association with the risk of adverse reactions, such as infection, phlebitis and deep venous thrombosis, for patients with solid malignancies receiving chemotherapy drugs (7-9). Nevertheless, the application of PICC for chemotherapy drugs is still relatively safe and effective (6). To ensure patient safety and increase the detection rate of venous complications, clinical observation alone is not sufficient. Therefore, conducting in-depth research on the microscopic level of blood vessels is important.

Vinorelbine, a semi-synthetic vinca alkaloid, may bind to tubulin and suppress mitotic microtubule polymerization (10). It is a potent chemotherapeutic drug for treating breast and 
non-small cell lung cancer (11-13). Although oral administration was approved for the clinical application of vinorelbine in 2006 due to convenience and the low risk of venous thrombosis, intravenous infusion exhibits higher efficacy (14). For example, the results of a phase II study demonstrated that patients with non-small cell lung cancer treated with oral vinorelbine exhibited good tolerance, but displayed limited overall survival time compared with those treated with intravenous vinorelbine (15). Although PICCs exhibit various advantages, the catheter is left in the vein for a long time, thus phlebitis often occurs due to the stimulation of the blood vessel wall by the catheter, the chemical stimulation of blood vessels via the drug infusion and the low obstruction in patients with cancer (1-3). Therefore, it is important to strengthen clinical observation and actively prevent, reduce or eliminate the occurrence of complications as much as possible. Certain patients with an invisible thrombus do not exhibit clinical symptoms, but present with abnormal pathological and laboratory indicators, despite thrombus formation. Therefore, to ensure patient safety and increase the detection rate of venous complications, clinical observation alone is not adequate. Thus, it is important to conduct in-depth research at the microscopic level of blood vessels. However, there is a lack of models with vinorelbine administration via PICC and a lack of evidence regarding pathological changes of PICC vein complications at different stages. The present study aimed to construct a rabbit model with vinorelbine administration via PICC to dynamically monitor phlebitis and thrombosis changes, which may provide a reference for early prediction, timely prevention and treatment of PICC-related chemotherapy complications.

\section{Materials and methods}

Animals. The present study was approved by the Ethics Committee of the School of Medicine, Jinhua Polytechnic (Jinhua, China; approval no. 2019017). In total, 48 healthy New Zealand rabbits (weight, 2.5-3.0 kg; age, 3-month-old) were provided by Jinhua Center of Laboratory Animals, Jinhua Food and Drug Inspection and Testing Institute (Jinhua, China), including 24 non-pregnant female rabbits and 24 male rabbits. The rabbits were adaptively fed for 1 week in the animal experimental center in single cages and randomly numbered. The housing conditions were as follows: $18^{\circ} \mathrm{C}$; humidity, $65 \%$; light/dark cycles, $12 / 12 \mathrm{~h}$; and with sufficient food and clean water. The animal experiments were performed in accordance with the Guide for the Care and Use of Laboratory Animals of the National Institutes of Health (ncbi.nlm.nih.gov/books/NBK54050/).

Construction of the PICC rabbit model. The 1.9 Fr x $50 \mathrm{~cm}$ single-lumen PICCs (Fr, unit of the circumference of the catheter) were purchased from Unijet. The lumen and outer wall of the catheter were rinsed with $15 \mathrm{ml}$ normal saline. Before use, the catheter was further rinsed with $5 \mathrm{ml}$ normal saline to remove bubbles. The rabbits were anesthetized via an intraperitoneal injection of $30 \mathrm{mg} / \mathrm{kg}$ sodium pentobarbital (3\%; Sigma-Aldrich; Merck KGaA). After successful anesthesia, the length of the PICC insertion was measured for each rabbit. To perform the measurement procedures, the rabbit was placed in a supine position, and keeping the rabbit's ears upright and parallel to the body at $180^{\circ}$, the length of the PICC insertion was determined $(21-26 \mathrm{~cm})$ by measuring the length from the puncture point (posterior auricular vein) to the most evident stop of the cardiac apex (Fig. 1A). Following skin preparation (Fig. 1B), disinfection (Fig. 1B) and puncture (Fig. 1C), the PICC tube was inserted into posterior auricular vein to the predetermined length (Fig. 1D). After checking patency and initial fixation, $0.5 \mathrm{ml}$ Lipiodol ${ }^{\circledR}$ (Jiangsu Hengrui Pharmaceutical Co., Ltd.) was intrathecally injected. Subsequently, whether the PICC tube reached the anterior vena cava was observed under X-ray fluoroscopy. Blood samples $(1.8 \mathrm{ml})$ were collected from the ear vein. After flushing the PICC tube with $10 \mathrm{ml}$ saline, the catheter was closed and fixed with a $3 \mathrm{M}$ applicator. To prevent accidental extubation from the rabbit ear, a recovery collar was placed on each animal so as not to affect the blood circulation or food intake.

Evaluation of PICC models. The criteria for evaluating the successful establishment of PICC models were as follows: i) The established animal model of PICC was successful with one puncture; ii) there was no local damage or bleeding in the puncture; iii) the end of the catheter was not twisted or folded under X-ray fluoroscopy; and iv) the PICC tube was placed in the anterior vena cava. As presented in Fig. 2, the transparent dot corresponded to the end of the catheter.

Animal grouping and modeling. The PICC model rabbits were randomly divided into the following eight groups $(n=6$ per group): i) Control group (PICC in place for 1 day); ii) 2nd day of PICC placement (received the first cycle of vinorelbine administration); iii) 3rd day of PICC placement; iv) 7th day of PICC placement; v) 14th day of PICC placement; vi) 21st day of PICC placement; vii) 23rd day of PICC placement (received the second cycle of vinorelbine administration); and viii) 24th day of PICC placement. Vinorelbine $(10 \mathrm{mg} / \mathrm{ml})$ was purchased from Jiangsu Haosoh Pharmaceutical Group Co., Ltd. After being dissolved in $10 \mathrm{ml}$ normal saline, vinorelbine was slowly injected into the rabbit using an infusion pump for $15 \mathrm{~min}$. The drug was administered once on the 2 nd day and once on the 23rd day after intubation. After each administration, the tube was sealed with heparin saline according to the pulse positive pressure method (16). Based on the Meeh-Rubner formula (17), the body surface area of each rabbit was calculated, and $25 \mathrm{mg} / \mathrm{m}^{2}$ was used as the optimal dose of vinorelbine. During the intermittent period of chemotherapeutics, PICC intubation maintenance was completed according to regular operations: The dressing was changed once a week; and when the dressing was wet or curled, it was disinfected and replaced. After the experiment, considering humane endpoints and animal welfare (when the rabbit experienced severe pain, suffering or dying or when the experiment was complete), all rabbits were euthanized via an intravenous injection of $100 \mathrm{mg} / \mathrm{kg}$ sodium pentobarbital to minimize suffering and distress. Death was verified by the complete cessation of the heartbeat and breathing and disappearance of reflexes.

General indicator observations. The local skin temperature, redness and extent of swelling at the puncture point were observed for the rabbits in each group. The criteria for evaluating the degree of phlebitis according to the phlebitis 
A
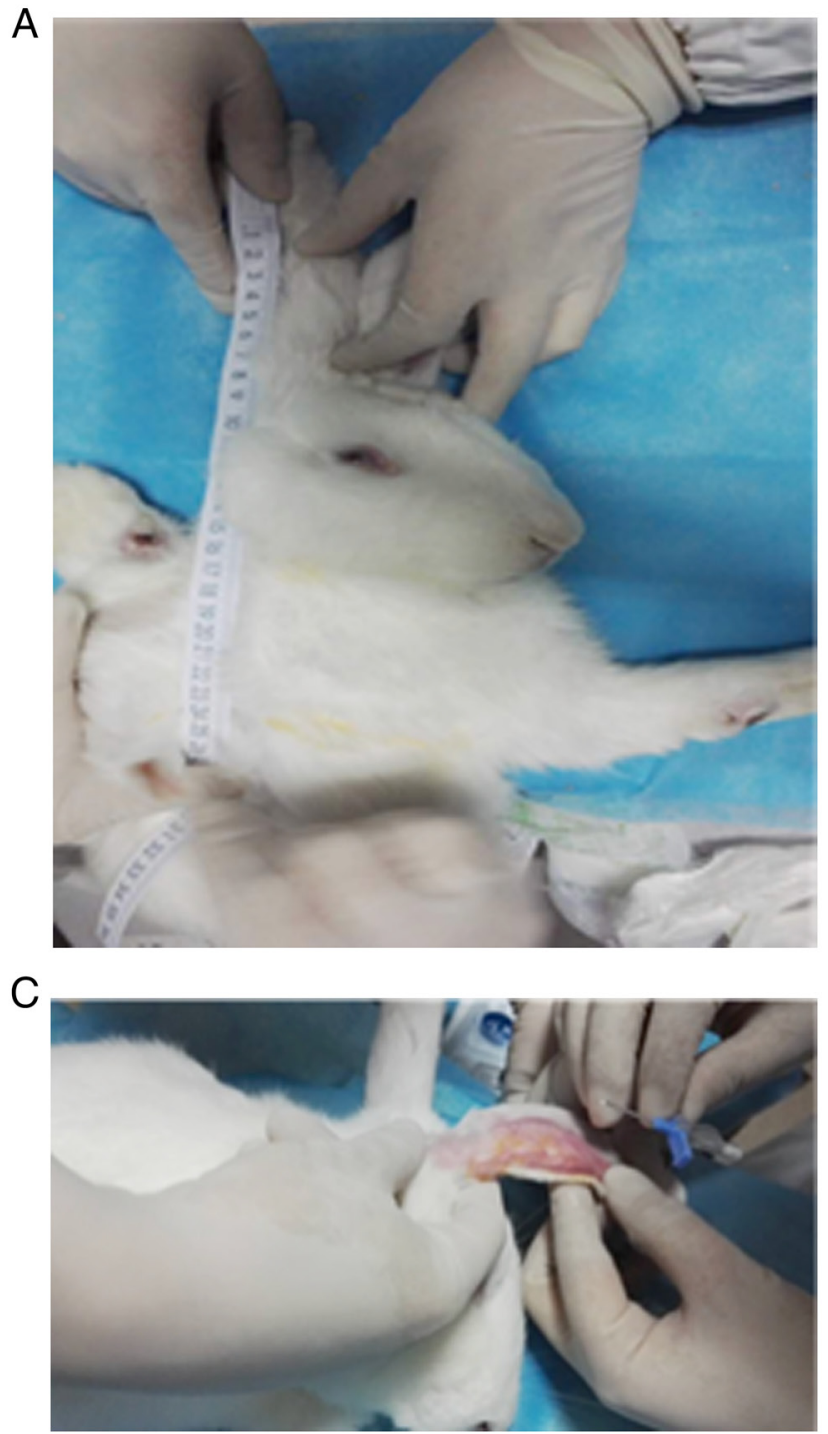

$\mathrm{B}$

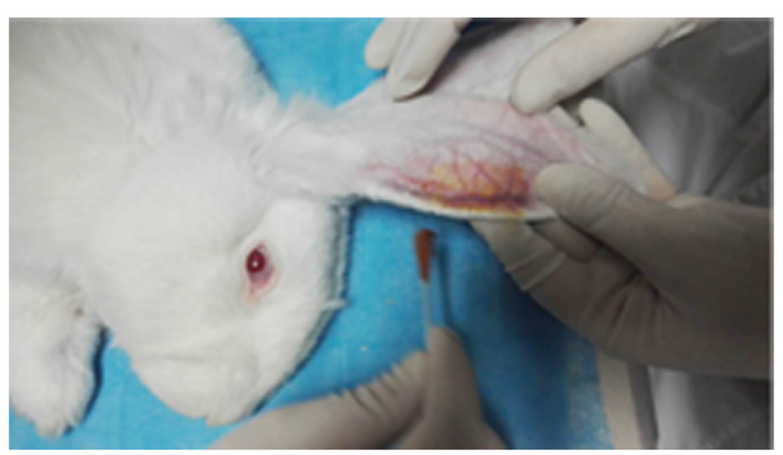

$\mathrm{D}$

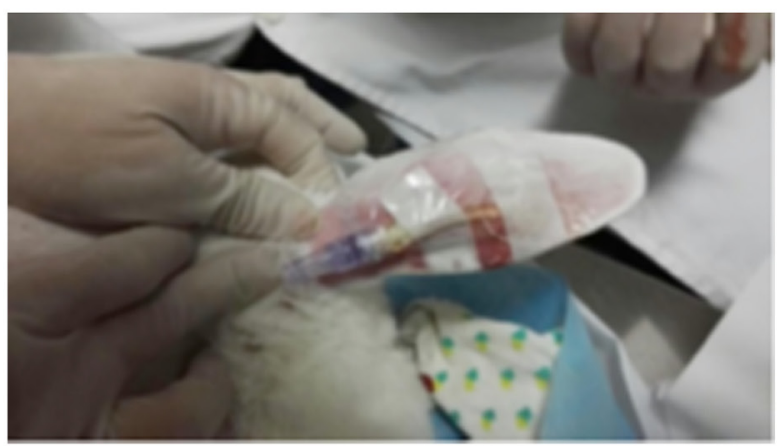

Figure 1. Construction of PICC chemotherapy rabbit model. (A) Measurement of the length of the PICC insertion. (B) Skin preparation and disinfection, (C) puncture and (D) PICC insertion. PICC, peripherally inserted central catheter.

grading standard of the American Society of Intravenous Nursing (18) were as follows: i) Grade 0, no clinical symptoms and signs; ii) grade I, local pain, redness or edema, no cord-like changes in the vein and no induration on touch; iii) grade II, local pain, redness, swelling or edema, string-like changes in veins, no induration, mild swelling, burning sensation and moderate pain; and iv) grade III, local pain, redness or edema, string-like changes in veins and palpable induration. Furthermore, venous thrombosis was evaluated based on the following indicators: i) Whether the puncture blood vessel infusion was unobstructed or not; ii) whether the local skin was swollen and displayed edema or not; and iii) the thrombus shape, color and composition according to pathological analysis.

Hematoxylin and eosin $(H \& E)$ staining. Following removal of the PICC tube, two sections $(5 \mathrm{~cm})$ were removed at the front and back of the catheter; furthermore, the ear vein and the anterior vena cava $(3 \mathrm{~cm})$ were removed. Ear vein, anterior vena cava and catheter samples were fixed with $10 \%$ formalin for $24 \mathrm{~h}$ at $4^{\circ} \mathrm{C}$. Following dehydration using an ethanol series, the samples were cleared using xylene I/II. Paraffin-embedded samples were sliced into 20,50, 100 or $200 \mu$ m-thick sections. Subsequently, H\&E staining was performed. Following dewaxing and rehydrating, the sections were stained with hematoxylin dye solution for $20 \mathrm{~min}$ at room temperature, followed by eosin staining for $1 \mathrm{~min}$ at room temperature. The sections were then dehydrated and cleared. After sealing with a neutral balsam, stained sections were visualized using a light microscope (Olympus Corporation; magnification, x15 or $\mathrm{x} 20$ ).

Prothrombin time. A total of $2 \mathrm{ml}$ ear vein blood samples were collected for measuring prothrombin time. Prothrombin time was determined using ACL-TOP700 automatic coagulation analyzer [ACL-TOP700; Wofen Medical Equipment Trading (Beijing) Co., Ltd.].

ELISA. Blood samples obtained from the ear vein were used for ELISA. Samples were maintained at room temperature for $2 \mathrm{~h}$. Subsequently, the samples were centrifuged at $1,000 \mathrm{xg}$ for $20 \mathrm{~min}$ at $4^{\circ} \mathrm{C}$ and then stored at $-20^{\circ} \mathrm{C}$. The serum levels of 


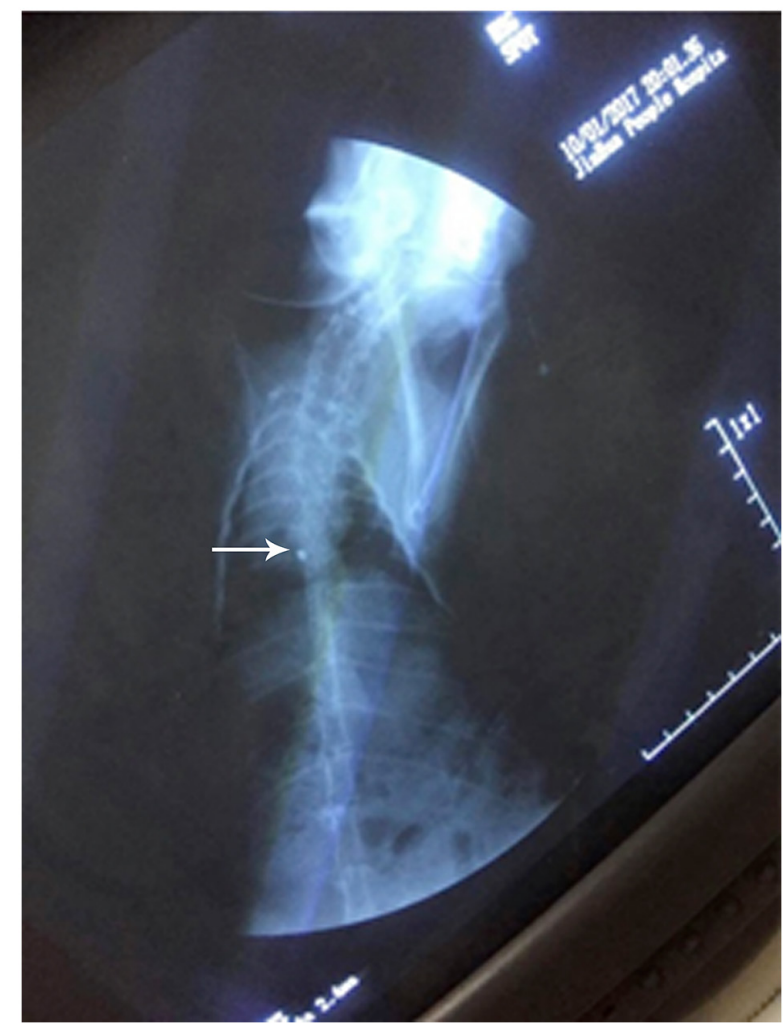

Figure 2. X-ray fluoroscopy of the PICC tube. X-ray demonstrated that the end of the PICC tube reached the anterior vena cava of the rabbit. Arrow indicates the transparent dot that highlights the end of the catheter. PICC, peripherally inserted central catheter.

E-selectin (cat. no. SEA029Rb; Cloud-Clone Corp.), P-selectin (cat. no. SEA569Ra), interleukin (IL)-2 (cat. no. SEA073Rb), IL-6 (cat. no. SEA079Rb), C-reactive protein (CRP; cat. no. SEA821Rb) and D-dimer (D2D; cat. no. CEA506Rb; all Cloud-Clone Corp.) were detected using the corresponding ELISA detection kits according to manufacturer's instructions. Optical density values were determined using a microplate reader (Bio-Rad Laboratories, Inc.).

Statistical analysis. Statistical analyses were performed using GraphPad Prism software (version 7.0; GraphPad Software, Inc.). Data are presented as the mean \pm standard deviation. Each experiment was repeated three times independently. Comparisons among multiple groups were performed using one-way ANOVA followed by Tukey's post hoc test. $\mathrm{P}<0.05$ was considered to indicate a statistically significant difference.

\section{Results}

Construction of a PICC chemotherapy rabbit model. In the present study, a PICC chemotherapy model was successfully established in 48 rabbits. To observe the pathological changes of the puncture point during PICC catheterization, the rabbits were randomly separated into eight experimental groups. A course of chemotherapy includes 2-3 cycles (19); the present study was based on two cycles. X-rays were captured to confirm that the end of the catheter was not twisted or folded, and was placed in the anterior vena cava (Fig. 3). In each group, the local puncture site displayed no injury or bleeding. Thus,

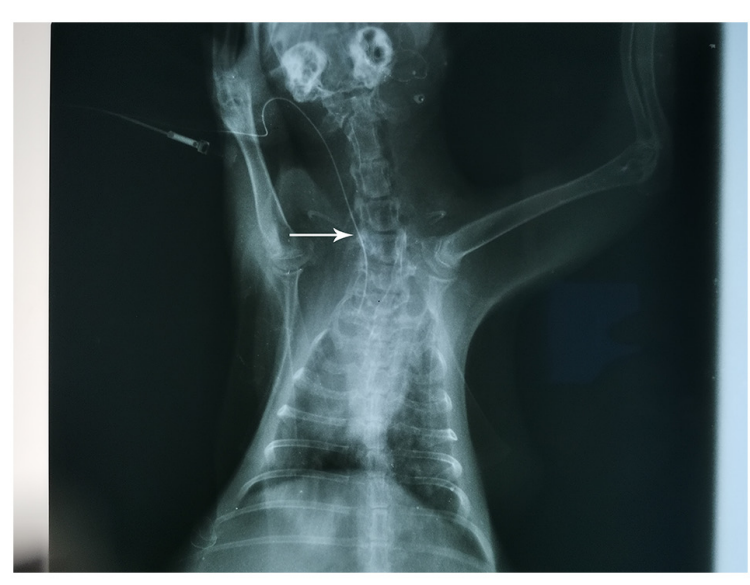

Figure 3. X-ray imaging determination of successful peripherally inserted central catheter model construction. The end of the catheter was not twisted or folded, and it was placed in the anterior vena cava. Arrow indicates the catheter.

it was determined that the PICC models were successfully constructed.

Dynamic monitoring of catheter-related thrombosis in rabbits with administration of vinorelbine via PICC. At different time periods, the PICC tube was removed and the two segments at the front and back of the catheter were surgically dissected. H\&E staining of the middle of the catheter was performed to observe catheter-related thrombosis. On the 1st day of PICC implantation, the H\&E staining results revealed that a thrombus filled the catheter (Fig. 4). On the 2nd day of PICC implantation, no thrombus was observed in the catheter after administration of vinorelbine. The catheter-related thrombus was continuously monitored on the $3 \mathrm{rd}$, 7th, 14th and 21st days of PICC implantation. As indicated by the H\&E staining results, there was no thrombus in the catheter. On the 23rd day of PICC implantation, a small thrombus was observed in the catheter. However, the thrombus was no longer visible on the 24th day. Collectively, the results indicated that catheter-related thrombosis primarily occurred on the 1st day of PICC implantation and the 23rd day of chemotherapy administration.

Dynamic monitoring of ear vein thrombosis after administration of vinorelbine via PICC. H\&E staining was performed to examine the pathological changes in ear vein tissues at numerous time points (Fig. 5). On the 1st day of PICC implantation, the intima of the ear veins was irregularly ruptured, and the lumen was characterized by thrombosis. On the 2nd day of catheterization, there was slight ear vein thrombosis, the intima was irregularly ruptured, and inflammatory cell infiltration was observed following vinorelbine administration. On the 3rd, 7th and 14th days of PICC implantation, ear vein thrombosis and inflammatory cell infiltration were notably improved, the intima was hyperplastic, and scars were formed in the lumen of the ear veins. Furthermore, the media and adventitia had no obvious lesions, and the lumen was not completely occluded. On the 21st and 23rd (after vinorelbine administration) days of PICC implantation, the results revealed that there were evident scars and inflammatory cell infiltration in the lumen. On the 24th day, the blood vessels of the 
Control

(1st day of PICC)

2nd day of PICC (+vinorelbine)

3rd day of PICC

7th day of PICC

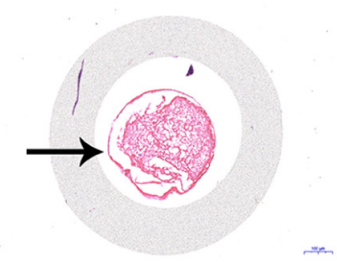

14th day of PICC

21st day of PICC

23rd day of PICC (+vinorelbine)

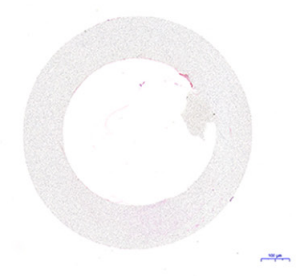

24th day of PICC
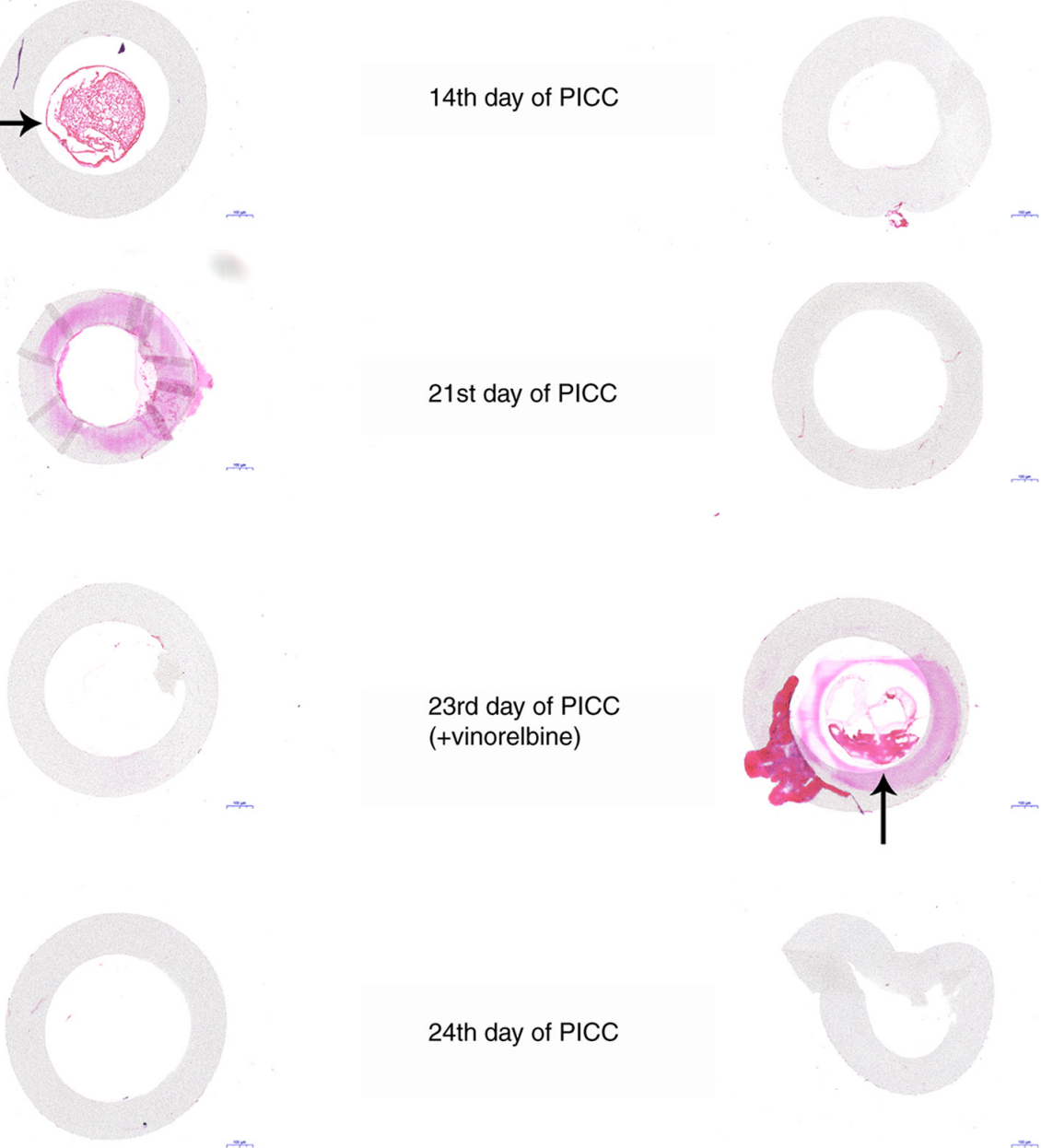

Figure 4. Dynamic monitoring of catheter-related thrombosis in rabbits with PICC administration of vinorelbine. Hematoxylin and eosin staining was performed to assess catheter-related thrombosis in rabbits with PICC placement at eight different time points. Arrows indicate the thrombus. Magnification, x15. Scale bar, $100 \mu \mathrm{m}$. PICC, peripherally inserted central catheter.

ear veins were not damaged, there was no obvious scar in the lumen and thrombosis had disappeared. Therefore, the results indicated that ear vein thrombosis was primarily caused by PICC puncture, and administration of vinorelbine may induce inflammatory cell infiltration.

Dynamic monitoring of pathological injury in the anterior vena cava after administration of vinorelbine via PICC. The end of the anterior vena cava was obtained at different time periods to investigate pathological injury via $\mathrm{H} \& \mathrm{E}$ staining. On the 1st day of PICC implantation, the vascular intima of the anterior vena cava was irregularly ruptured (Fig. 6). Furthermore, the vessel wall was thickened, which was accompanied by immune cell infiltration. On the 2nd (after vinorelbine administration), 3rd and 7th days of PICC implantation, the vascular intima of the anterior vena cava was relatively intact, with a slightly thickened wall. Moreover, inflammatory cell infiltration was distinctly ameliorated. On the 14th and 21st days, the vascular intima of the anterior vena cava was intact and the wall was normal. However, low level immune cell infiltration was observed. On the 23rd day of PICC implantation after administration of vinorelbine, there was distinct inflammatory cell infiltration in the anterior vena cava. On the 24th day, the vascular intima of the anterior vena cava was relatively intact, the wall was normal and there was almost no infiltration of inflammatory cells. These results indicated that vinorelbine administered via PICC could induce phlebitis, and over time phlebitis was gradually ameliorated within the first cycle of vinorelbine and phlebitis was significantly alleviated within 1 day of the second cycle of vinorelbine

Evaluation of prothrombin time after administration of vinorelbine via PICC. To assess the function of the extrinsic coagulation system, the prothrombin time of ear vein blood samples that were collected at different time points was assessed. The results demonstrated that the prothrombin time was significantly shortened after administration of vinorelbine on the 2nd day of PICC implantation compared with that on the 1st day (Fig. 7). Compared with that on the 2nd day of PICC implantation with vinorelbine administration, the prothrombin time was significantly prolonged on the 7 th, 14 th and 21 st days. On the 23rd day, after administration of vinorelbine, the prothrombin time was significantly reduced compared with that on the 21st day. By contrast, the prothrombin time was significantly increased on the 24th day compared with that on the 23rd day. Therefore, the results indicated that administration of vinorelbine via PICC reduced prothrombin time on the day of administration. 


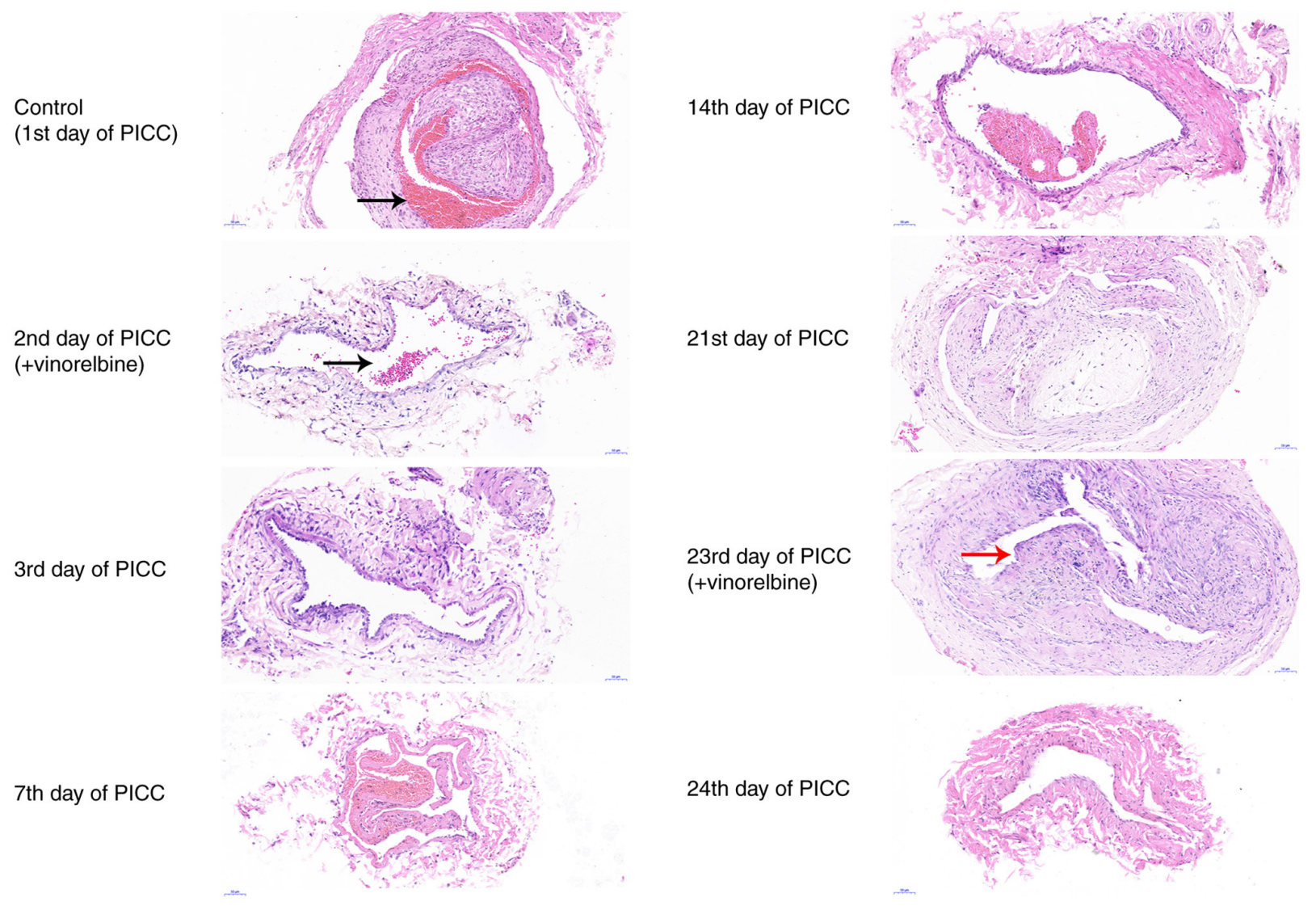

Figure 5. Dynamic monitoring of ear vein thrombosis in rabbits with PICC administration of vinorelbine. Hematoxylin and eosin staining was performed to assess the ear vein thrombosis in rabbits with PICC placement at eight different time points. Black arrows indicate ear vein thrombosis. Red arrow indicates inflammatory cell infiltration. Magnification, x20. Scale bar, $50 \mu \mathrm{m}$. PICC, peripherally inserted central catheter.

Dynamic monitoring of inflammation- and thrombosis-related factors in rabbits administered with vinorelbine via PICC. Inflammation- and thrombosis-related indexes were examined in blood samples obtained from ear veins via ELISA at different time points. The levels of CRP after administration of vinorelbine on the 2nd day of PICC implantation were significantly increased compared with those on the 1st day of catheterization (Fig. 8A). However, on the 7th, 14th and 21st days of PICC implantation, CRP levels were significantly lowered compared with those on the 2nd day. On the 23rd day of PICC implantation after vinorelbine administration, CRP levels were significantly elevated compared with those on the 21st day, but this effect was significantly reversed on the 24th day. The levels of P-selectin, E-selectin and IL-6 were also determined in ear vein blood samples. P-selectin, E-selectin and IL-6 levels were significantly higher on the 2nd day of PICC after vinorelbine administration compared with those on the 1st day of catheterization (Fig. 8B-D). Nevertheless, the levels of these markers were significantly decreased on the 7th, 14th and 21st days of PICC implantation compared with those on the 2nd day. Following administration of vinorelbine on the 23rd day of catheterization, P-selectin, E-selectin, and IL-6 levels were significantly elevated compared with those on the 21st day, but this effect was reversed on the 24th day. As demonstrated in Fig. 8E, IL-2 levels were significantly reduced after vinorelbine administration on the 2nd day of PICC catheterization compared with those on the 1st day of catheterization. On the 7th, 14th and 21st days, IL-2 levels were significantly increased in a time-dependent manner compared with those on the 2nd day. After administration of vinorelbine on the 23rd day of catheterization, IL-2 levels were significantly reduced compared with those on the 21st day, but then significantly increased on the 24th day. D2D levels were significantly higher on the 2nd day of PICC implantation after vinorelbine administration compared with those on the 1st day of PICC (Fig. 8F). On the 7th, 14th and 21st days, D2D levels were significantly decreased compared with those on the 2nd day. However, significantly increased D2D levels were detected following administration of vinorelbine on the 23rd day compared with those on the 21st day. The levels of D2D displayed a significant decline on the 24th day compared with those on the 23rd day. These results revealed that vinorelbine administration via PICC could induce an inflammatory response and thrombosis formation.

\section{Discussion}

In the present study, a rabbit model with vinorelbine administration via PICC was successfully established. During each time point of PICC intubation, the puncture points, vascular pathological changes and serum observation indexes were assessed. Eight time points of PICC intubation were used to observe phlebitis and venous thrombosis. Administration of vinorelbine via PICC distinctly induced catheter-related thrombosis, ear vein thrombosis and pathological damage in the anterior vena cava. Furthermore, prothrombin time was 


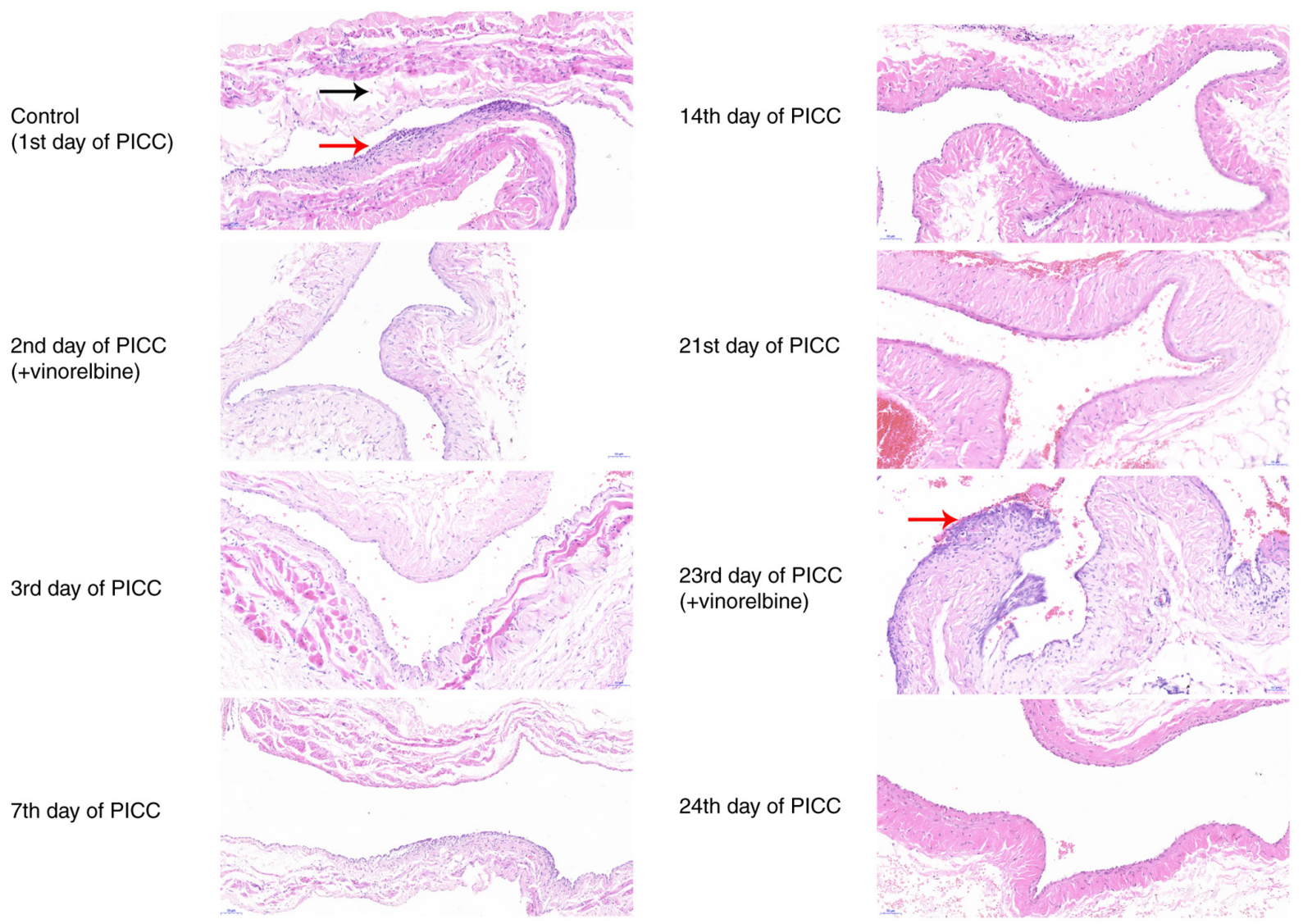

Figure 6. Dynamic monitoring of pathological injury in the anterior vena cava in rabbits with PICC administration of vinorelbine. Hematoxylin and eosin staining was performed to assess the pathological changes in the anterior vena cava tissues in rabbits with PICC placement at eight different time points. Black arrow indicates irregularly ruptured vascular intima. Red arrows indicate immune cell infiltration. Magnification, x20. Scale bar, $50 \mu \mathrm{m}$. PICC, peripherally inserted central catheter.

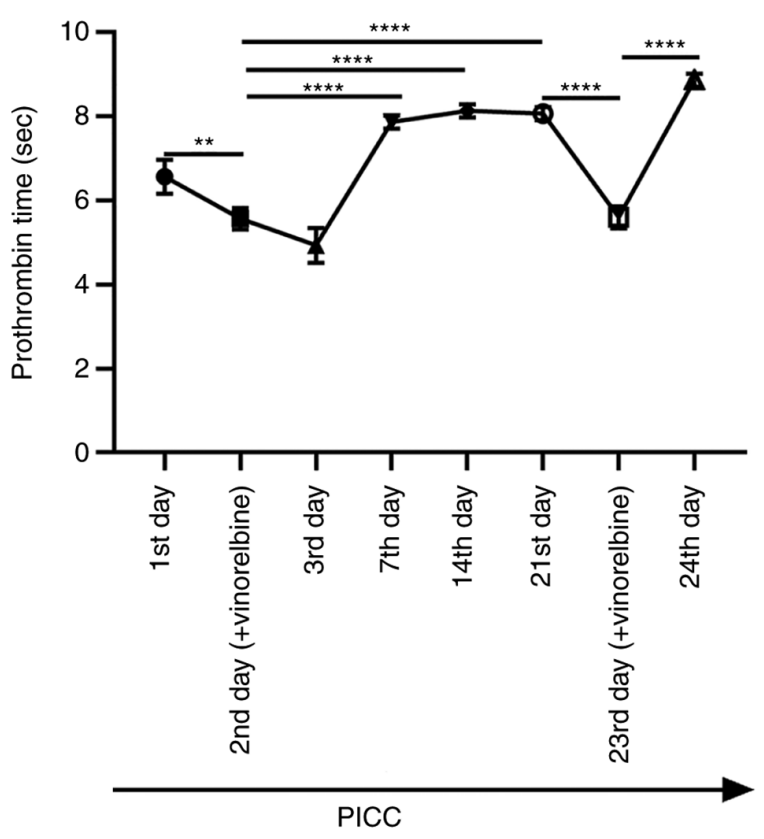

Figure 7. Prothrombin time of ear vein blood samples in rabbits with PICC placement. Prothrombin time was assessed at eight different time points. ${ }^{* * *} \mathrm{P}<0.01 ;{ }^{* * * *} \mathrm{P}<0.0001$. PICC, peripherally inserted central catheter.

significantly decreased, and the inflammatory response was significantly enhanced after vinorelbine administration via
PICC. With increasing time after PICC administration of vinorelbine, the aforementioned pathological changes were notably improved.

$\mathrm{X}$-rays confirmed that the PICC rabbit model was successfully constructed in the present study. For patients with cancer receiving chemotherapy or nutrition, PICC has become the main method of venous access (20-22). However, PICC-related venous thrombosis is the most common complication (23). For example, it has been reported that PICC is associated with a high risk of catheter-related deep venous thrombosis in a randomized multicenter trial (3). Consistently, the results of the present study revealed that the catheter was filled with a thrombus on the 1st day of PICC implantation. However, after administration of $25 \mathrm{mg} / \mathrm{m}^{2}$ vinorelbine on the $2 \mathrm{nd}$ and $23 \mathrm{rd}$ day of PICC, the vein thrombus was observed. Furthermore, pathological damage in the anterior vena cava was relatively minimal on the day of vinorelbine administration. However, with increasing time the pathological damage was gradually ameliorated. In a recent monocentric and randomized trial, PICC displayed higher safety and effectiveness compared with a centrally inserted central catheter. Moreover, the use of PICC could effectively reduce the risk of infection and thrombosis (24). However, whether vinorelbine injection via PICC is effective and safe requires long-term investigation in a larger cohort.

Prothrombin time refers to the time required to add excessive tissue thromboplastin and calcium ions to plasma lacking 

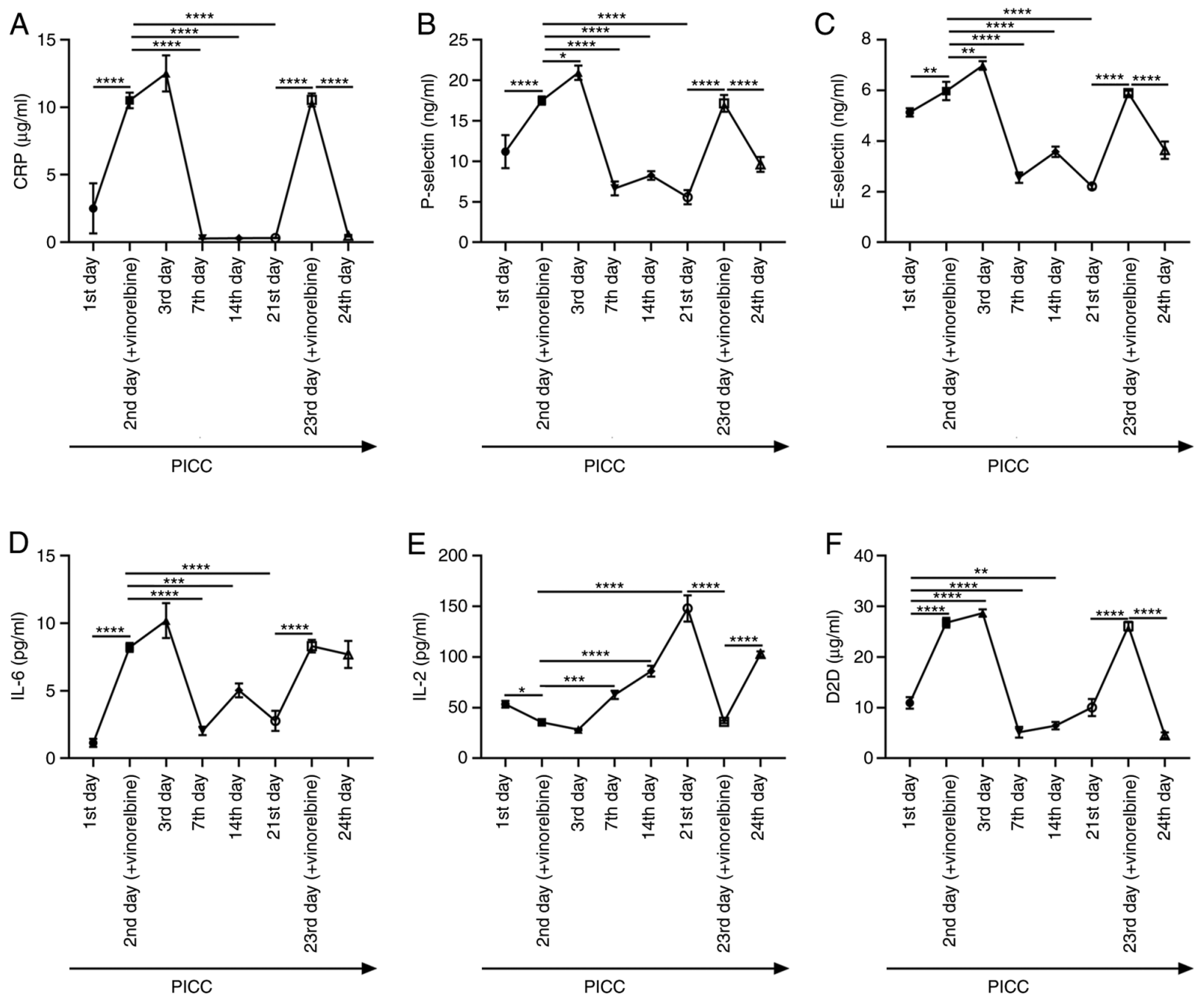

Figure 8. Inflammation- and thrombosis-related factors in rabbits with PICC administration of vinorelbine. Levels of (A) CRP, (B) P-selectin, (C) E-selectin, (D) IL-6, (E) IL-2 and (F) D2D were determined in ear vein blood samples from rabbits with PICC placement at eight different time points by performing ELISA. ${ }^{*} \mathrm{P}<0.05,{ }^{* * *} \mathrm{P}<0.01,{ }^{* * *} \mathrm{P}<0.001$ and ${ }^{* * * * *} \mathrm{P}<0.0001$. PICC, peripherally inserted central catheter; CRP, C-reactive protein; IL, interleukin; D2D, D-dimer.

platelets to convert prothrombin to thrombin, resulting in plasma coagulation (25-27). Prothrombin time is an indicator reflecting the activity of coagulation factors I, II, V, VII and $\mathrm{X}$ in the plasma (28). The prothrombin time measurement is a barrier-free screening test to check the function of the extrinsic coagulation system, and it is also an important monitoring index for clinical anticoagulation therapy (29). In the present study, compared with that on the 1st day of PICC, the prothrombin time was significantly shortened following vinorelbine administration, but significantly increased over time. In a previous study, a positive correlation between serum vinorelbine levels and the number of platelets was reported (30).

Serum inflammation- and thrombosis-related factors were examined by performing ELISA. The results demonstrated that CRP, P-selectin, E-selectin, IL-6 and D2D levels were distinctly elevated following vinorelbine administration. CRP, which is a non-specific diagnostic inflammatory biomarker, is involved in mediating innate immune responses (31). The levels of CRP in the plasma rise sharply when the body is infected or tissues are damaged (32). In the early stage of acute inflammation, P-selectin participates in the process of recruiting leukocytes to the injured site (33). Moreover, P-selectin is closely related to deep vein thrombosis (34). D2D is a fibrin degradation product, and its elevated levels indicate a hypercoagulable state and secondary fibrinolysis in the body (35). It has become a diagnostic marker for deep vein thrombosis (36). In the present study, administration of vinorelbine via PICC significantly elevated serum CRP, P-selectin, E-selectin, IL-6 and D2D levels but decreased serum IL-2 levels, indicating that inflammation and thrombosis could be induced by vinorelbine administration via PICC. A previous study demonstrated that vinorelbine reduces serum IL-2 levels in a Lewis lung cancer mouse model (37).

However, the present study had certain limitations. Firstly, to dynamically observe phlebitis and venous thrombosis, additional time points should be assessed. Moreover, the potential underlying mechanisms should be explored in future studies.

The present study successfully constructed a rabbit model with vinorelbine administration via PICC, and dynamically observed phlebitis and venous thrombosis. On the day of vinorelbine administration via PICC, there was a high risk of phlebitis and thrombosis, which suggested that anticoagulation therapy and patient care should be provided in that time period. Therefore, the present study provided a reference for early 
prediction, timely prevention and treatment of PICC-related chemotherapy venous complications, and also provided a theoretical basis for timely vascular protection, anticoagulation therapy and effective patient care.

\section{Acknowledgements}

Not applicable.

\section{Funding}

The present study was supported by the Zhejiang Province Experimental Animal Science and Technology Planning Project in 2018 (grant no. 2018C37095).

\section{Availability of data and materials}

The datasets used and/or analyzed during the current study are available from the corresponding author on reasonable request.

\section{Authors' contributions}

LH conceived and designed the study. GC and QH performed the majority of experiments and data analysis, and wrote the manuscript. $\mathrm{BH}, \mathrm{LZ}$ and LF performed a small number of experiments and data analysis, and wrote and revised the manuscript. LH and GC confirm the authenticity of all the raw data. All authors have read and approved the final manuscript.

\section{Ethics approval and consent to participate}

The present study was approved by the Ethics Committee of the School of Medicine, Jinhua Polytechnic (Jinhua, China; approval no. 2019017).

\section{Patient consent for publication}

Not applicable.

\section{Competing interests}

The authors declare that they have no competing interests.

\section{References}

1. Keren R, Shah SS, Srivastava R, Rangel S, Bendel-Stenzel M, Harik N, Hartley J, Lopez M, Seguias L, Tieder J, et al: Comparative effectiveness of intravenous vs oral antibiotics for postdischarge treatment of acute osteomyelitis in children. JAMA Pediatr 169: 120-128, 2015.

2. Campagna S, Gonella S, Berchialla P, Morano G, Rigo C, Zerla PA, Fuzzi R, Corona G, Storto S, Dimonte V and Mussa B: Can peripherally inserted central catheters be safely placed in patients with cancer receiving chemotherapy? A retrospective study of almost 400,000 catheter-days. Oncologist 24: e953-e959, 2019.

3. Taxbro K, Hammarskjöld F, Thelin B, Lewin F, Hagman H, Hanberger $\mathrm{H}$ and Berg S: Clinical impact of peripherally inserted central catheters vs implanted port catheters in patients with cancer: An open-label, randomised, two-centre trial. $\mathrm{Br}$ J Anaesth 122: 734-741, 2019.

4. Yu D, Zhang K, Huang L, Zhao B, Zhang X, Guo X, Li M, Gu Z, Fu G, Hu M, et al: Detection of peripherally inserted central catheter (PICC) in chest X-ray images: A multi-task deep learning model. Comput Methods Programs Biomed 197: 105674, 2020.
5. Jumani K, Advani S, Reich NG, Gosey L and Milstone AM: Risk factors for peripherally inserted central venous catheter complications in children. JAMA Pediatr 167: 429-435, 2013.

6. Robinson A, Souied O, Bota AB, Levasseur N, Stober C, Hilton J, Kamel D, Hutton B, Vandermeer L, Mazzarello S, et al: Optimal vascular access strategies for patients receiving chemotherapy for early-stage breast cancer: A systematic review. Breast Cancer Res Treat 171: 607-620, 2018.

7. Fallouh N, McGuirk HM,Flanders SA and Chopra V: Peripherally inserted central catheter-associated deep vein thrombosis: A narrative review. Am J Med 128: 722-738, 2015.

8. Lv S, Liu Y, Wei G, Shi X, Chen S and Zhang X: The anticoagulants rivaroxaban and low molecular weight heparin prevent PICC-related upper extremity venous thrombosis in cancer patients. Medicine (Baltimore) 98: e17894, 2019.

9. Pu YL, Li ZS, Zhi XX, Shi YA, Meng AF, Cheng F, Ali A, Li C, Fang $\mathrm{H}$ and Wang $\mathrm{C}$ : Complications and costs of peripherally inserted central venous catheters compared with implantable port catheters for cancer patients: A meta-analysis. Cancer Nurs 43: 455-467, 2020.

10. Perri F, Lazzari G, Scarpati GD and Silvano G: Oral vinorelbine: A feasible and safe partner for radiotherapy in the treatment of locally advanced non-small cell lung cancer. Onco Targets Ther 9: 2359-2364, 2016.

11. Diaby V, Tawk R, Sanogo V, Xiao H and Montero AJ: A review of systematic reviews of the cost-effectiveness of hormone therapy, chemotherapy, and targeted therapy for breast cancer. Breast Cancer Res Treat 151: 27-40, 2015.

12. Harbeck N, Huang CS, Hurvitz S, Yeh DC, Shao Z, Im SA, Jung KH, Shen K, Ro J, Jassem J, et al: Afatinib plus vinorelbine versus trastuzumab plus vinorelbine in patients with HER2-overexpressing metastatic breast cancer who had progressed on one previous trastuzumab treatment (LUX-Breast 1): An open-label, randomised, phase 3 trial. Lancet Oncol 17: 357-366, 2016.

13. Mondal A, Gandhi A,Fimognari C, Atanasov AG and Bishayee A: Alkaloids for cancer prevention and therapy: Current progress and future perspectives. Eur J Pharmacol 858: 172472, 2019.

14. Jonna S, Reuss JE, Kim C and Liu SV: Oral chemotherapy for treatment of lung cancer. Front Oncol 10: 793, 2020.

15. Rossi D, Catalano V, Alessandroni P, Fedeli A, Fedeli SL, Giordani P, Baldelli AM, Casadei V, Ceccolini M, Ugolini M, et al: A phase II study of single-agent oral vinorelbine in patients with pretreated advanced non-small-cell lung cancer. Clin Lung Cancer 8: 382-385, 2007.

16. Markert JE, Jasien JV, Turner DC, Huisingh C, Girkin CA and Downs JC: IOP, IOP transient impulse, ocular perfusion pressure, and mean arterial pressure relationships in nonhuman primates instrumented with telemetry. Invest Ophthalmol Vis Sci 59: 4496-4505, 2018.

17. Ohwada K: Body surface area of the golden Syrian hamster. Jikken Dobutsu 41: 221-224, 1992.

18. Oragano CA, Patton D and Moore Z: Phlebitis in intravenous amiodarone administration: Incidence and contributing factors. Crit Care Nurse 39: e1-e12, 2019.

19. Wilson BE, Jacob S, Yap ML, Ferlay J, Bray F and Barton MB: Estimates of global chemotherapy demands and corresponding physician workforce requirements for 2018 and 2040: A population-based study. Lancet Oncol 20: 769-780, 2019.

20. Bertoglio S, Cafiero F, Meszaros P, Varaldo E, Blondeaux E, Molinelli C and Minuto M: PICC-PORT totally implantable vascular access device in breast cancer patients undergoing chemotherapy. J Vasc Access 21: 460-466, 2020.

21. Bertoglio S, Faccini B, Lalli L, Cafiero F and Bruzzi P: Peripherally inserted central catheters (PICCs) in cancer patients under chemotherapy: A prospective study on the incidence of complications and overall failures. J Surg Oncol 113: 708-714, 2016.

22. Qi F, Cheng H, Yuan X and Zhang L: Comparison of PICC and TIVAP in chemotherapy for patients with thyroid cancer. Oncol Lett 20: 1657-1662, 2020.

23. Wang G, Li Y, Wu C, Guo L, Hao L, Liao H, Xiao X, Liu S and Luo L: The clinical features and related factors of PICC-related upper extremity asymptomatic venous thrombosis in cancer patients: A prospective study. Medicine (Baltimore) 99: e19409, 2020.

24. Picardi M, Della Pepa R, Cerchione C, Pugliese N, Mortaruolo C, Trastulli F, Giordano C, Grimaldi F, Zacheo I, Raimondo M, et al: A frontline approach with peripherally inserted versus centrally inserted central venous catheters for remission induction chemotherapy phase of acute myeloid leukemia: A randomized comparison. Clin Lymphoma Myeloma Leuk 19: e184-e194, 2019. 
25. Ng VL: Prothrombin time and partial thromboplastin time assay considerations. Clin Lab Med 29: 253-263, 2009.

26. Tripodi A: Prothrombin time international normalized ratio monitoring by self-testing. Curr Opin Hematol 11: 141-145, 2004.

27. Yang DT, Robetorye RS and Rodgers GM: Home prothrombin time monitoring: A literature analysis. Am J Hematol 77: 177-186, 2004.

28. Naghadeh HT, Maghsudloo M and Tabatabaei MR: Coagulation factors V, VIII, and X, prothrombin time and activated partial thromboplastin time test results in thawed plasma stored at $1-6^{\circ} \mathrm{C}$ for 5 days. Blood Transfus 9: 95-98, 2011.

29. Onundarson PT, Francis CW, Indridason OS, Arnar DO, Bjornsson ES, Magnusson MK, Juliusson SJ, Jensdottir HM, Vidarsson B, Gunnarsson PS, et al: Fiix-prothrombin time versus standard prothrombin time for monitoring of warfarin anticoagulation: A single centre, double-blind, randomised, non-inferiority trial. Lancet Haematol 2: e231-240, 2015.

30. Gusella M, Pasini F, Caruso D, Barile C, Modena Y, Fraccon AP, Bertolaso L, Menon D, Crepaldi G, Bononi A, et al: Clinical outcomes of oral metronomic vinorelbine in advanced non-small cell lung cancer: Correlations with pharmacokinetics and MDR1 polymorphisms. Cancer Chemother Pharmacol 83: 493-500, 2019.

31. Yao Z, Zhang $\mathrm{Y}$ and $\mathrm{Wu} \mathrm{H}$ : Regulation of $\mathrm{C}$-reactive protein conformation in inflammation. Inflamm Res 68: 815-823, 2019.

32. Pathak A and Agrawal A: Evolution of C-reactive protein. Front Immunol 10: 943, 2019.
33. Setiadi H, Yago T, Liu Z and McEver RP: Endothelial signaling by neutrophil-released oncostatin M enhances P-selectin-dependent inflammation and thrombosis. Blood Adv 3: 168-183, 2019.

34. Wei Y, Chen X, Shen H, Wu W, Cao G, Chen W, Wang Y, Shen H, Yu S and Zhang J: P-selectin level at first and third day after portal hypertensive splenectomy for early prediction of portal vein thrombosis in patients with cirrhosis. Clin Appl Thromb Hemost 24 (9_Suppl): 76S-83S, 2018.

35. Riva N, Camporese G, Iotti M, Bucherini E, Righini M, Kamphuisen PW, Verhamme P, Douketis JD, Tonello C, Prandoni P, et al: Age-adjusted D-dimer to rule out deep vein thrombosis: Findings from the PALLADIO algorithm. J Thromb Haemost 16: 271-278, 2018.

36. Wells PS, Anderson DR, Rodger M, Forgie M, Kearon C, Dreyer J, Kovacs G, Mitchell M, Lewandowski B, Kovacs MJ, et al: Evaluation of D-dimer in the diagnosis of suspected deep-vein thrombosis. N Engl J Med 349: 1227-1235, 2003.

37. Orlandi P, Banchi M, Alì G, Desidero TD, Fini E, Fontanini G and Bocci G: Active metronomic vinorelbine schedules decrease plasma interleukin-2 levels in mice with Lewis lung carcinoma. J Chemother 33: 198-202, 2021.

This work is licensed under a Creative Commons Attribution-NonCommercial-NoDerivatives 4.0 International (CC BY-NC-ND 4.0) License. 